\title{
Mixing of shear Alfven wave packets
}

\author{
N. Bian ${ }^{1}$ and D. Tsiklauri ${ }^{2}$ \\ 1 Jodrell Bank Centre for Astrophysics, School of Physics and Astronomy, University of Manchester, Manchester, M60 1QD, UK \\ e-mail: nbian@hotmail.com \\ 2 Institute for Material Research, University of Salford, Greater Manchester, M5 4WT, UK
}

Received 21 September 2007 / Accepted 26 May 2008

\section{ABSTRACT}

\begin{abstract}
Aims. We consider the propagation of shear Alfven wave packets in inhomogeneous magnetic fields which is at the origin of their distortion regardless of any non-linear coupling. It is shown that this can be regarded as a mixing process and hence, standard phase mixing corresponds to the effect of an Alfvenic shear flow, while enhanced dissipation at a magnetic X-point corresponds to mixing by an Alfvenic strain flow.

Methods. The evolution of the wave field is supposed to result from the dynamics of a superposition of wave packets, and a kinetic equation for the wave energy is obtained following this eikonal (WKB) description.

Results. Since shear Alfven wave packets experience continuous shearing/straining, while transported by an inhomogeneous Alfvenic flow $\boldsymbol{V}_{\mathrm{A}}$, their mixing process in physical space is also a cascade of wave energy in $k$-space. The wave energy spectrum resulting from this linear mechanism of energy transfer is determined for the special case of waves propagating along chaotic magnetic field lines, the analog of a chaotic mixing process. The latter follows a $k^{-1}$ power law in the energy conserving range in $k$ space.
\end{abstract}

Key words. magnetohydrodynamics (MHD) - waves - magnetic fields

\section{Introduction}

Phase mixing, i.e. the propagation and enhanced dissipation of shear Alfven waves, remain active areas of research in studies of the solar corona, in relation both to its heating mechanism and the acceleration of the fast solar wind; see e.g. reviews on the subjects (Browning 1989; Walsh \& Ireland 2003; Ofman 2005) and references within. The reason is that if Alfven waves are excited and dissipate some of their energy into the solar corona, this contributes to the overall coronal energy budget.

Because of the very low collisionality of the medium, a main difficulty of wave dissipation theories is, however, to find an explanation of how these fluctuations can thermalize, i.e. reach the very small dissipative scale, before they leave the corona. But since the coronal plasma is highly inhomogeneous, it is quite natural to invoke wave interaction with the ambient background as a reason for the creation of fine enough scales in the wave field. It is in this context that phase mixing of Alfven waves was proposed by Heyvaerts \& Priest (1983) as an enhanced dissipation mechanism. They considered simple inhomogeneity of the medium, in one direction perpendicular to the unidirectional background magnetic field. The existence of a shear in the Alfven velocity $\boldsymbol{V}_{\mathrm{A}}$ results in progressively smaller transverse scales in the wave field while it propagates and is transported along the unperturbed magnetic field. In this case, the build-up of a diffusive scale is linear in time $(\mathrm{d} k / \mathrm{d} t \propto$ const.), resulting in a dissipation time proportional to $S^{1 / 3} \ll S$. Here, $S$ is the high Lundquist number $S \equiv \tau_{\eta} / \tau_{\mathrm{A}} \gg 1$ of the plasma. The corresponding diffusive scale is $S^{-1 / 3} \ll 1$. Therefore, the dissipation time is found to be much shorter than the very large diffusion time $\tau_{\eta}$, proportional to $S \gg 1$, for the wave to dissipate in the absence of inhomogeneity.

More recent studies have shown that the creation of a dissipative scale can be made exponentially fast $(\mathrm{d} k / \mathrm{d} t \propto$ const. $\cdot k)$, with the associated time scale being proportional to $\ln S \ll S^{1 / 3} \ll S$ (Similon \& Sudan 1989; Petkaki et al. 1998; Malara et al. 2007). The latter improved efficiency of the dissipation results by essentially considering a more complex magnetic geometry, with the property of local exponential separation or divergence of neighboring magnetic field lines. In this case, the diffusive length scale is proportional to $S^{-1 / 2}$. Therefore, fast dissipation is possible in three-dimensional chaotic magnetic fields (Similon \& Sudan 1989; Petkaki et al. 1998; Malara et al. 2000, 2007) and in twodimensional regular ones (Smith et al. 2007; Malara et al. 2003; Ruderman et al. 1998), provided they possess a degree of complexity.

One main goal of this work is to show that the propagation and distortion of shear Alfven wave packets in inhomogeneous magnetic fields can be regarded as a mixing effect. As is well known, the kinematics of mixing are governed by the topological properties of the flow. It naturally follows that the distortion of Alfven wave packets propagating in complex magnetic fields is the counterpart of a chaotic mixing process (Ottino 1988).

The interaction among shear Alfven waves is also a wellstudied mechanism of energy transfer onto small scales, the non-linear distortion resulting from elastic collisions between counter-propagating wave packets producing a flux of their energy to small dissipative scales. Nevertheless, linear distortion through interaction with the background produces a similar flux. In the following, we neglect non-linearities in the magnetohydrodynamics equations in order to focus primarily on the spectral properties of this energy transfer for waves propagating in complex magnetic fields.

We obtain a kinetic equation for the wave energy from a representation of the global wave perturbation as an ensemble of wave packets (Similon \& Sudan 1989). Hence, the wave energy spectrum $F(k, t)$ is the sum of the energy of each individual wave packet, with associated wave vector and position, whose 
time dependence is given by Hamiltonian ray equations. This WKB method has already been employed by several authors (Petkaki et al. 1998; Malara et al. 2000, 2007) to study shear Alfven waves propagation and dissipation in different types of coronal magnetic structures. Our study is a continuation of these works. In Sect. 4, we determine the transient and stationary energy spectra, $F(k, t)$ and $F(k)$, for the special case of noninteracting Alfven waves propagating in a synthetic chaotic magnetic configuration with a quasi-uniform rate of field-lines exponentiation (Similon \& Sudan 1989). In Sect. 3, we show the correspondence that exists between the problem of the enhanced dissipation of Alfven waves, transported by inhomogeneous Alfvenic flows, and the problem of mixing (Ottino 1988), reviewed in Sect. 2. Conclusions are given in the last section of this article.

\section{Mixing of a passive tracer}

The mixing of a passive field $\psi$ advected by a flow $\boldsymbol{U}$ is described by the linear advection-diffusion equation

$$
\frac{\partial \psi}{\partial t}+\boldsymbol{U} \cdot \nabla \psi=\eta \nabla^{2} \psi
$$

In absence of flow, $\boldsymbol{U}=0$, the field $\psi$ only diffuses on a large time scale for small diffusion coefficient $\eta$ because this time is proportional to $\eta^{-1}$. If the flow has some spatial variation, i.e. it is inhomogeneous, the advected field is stirred. It then develops increasing gradients, until diffusion takes over and ultimately mixes the field. Because diffusion alone is slow to mix, a stirring flow is required to accelerate the dissipative process. An important issue is therefore to find flows $\boldsymbol{U}$ that have the property of being good mixers. For the enhanced dissipation of shear-Alfven waves, this property concerns the Alfvenic flow $\boldsymbol{V}_{\mathrm{A}}$, which by definition is responsible for the advection of the wave field. To show this, we first concentrate on two standard and simple flow configurations having their counterpart in the problem of dissipation of Alfven waves, and compare their mixing efficiency.

Consider the effect of shear flow $\boldsymbol{U}=\left(U_{0}^{\prime} y, 0,0\right)$,

$$
\frac{\partial \psi}{\partial t}+U_{0}^{\prime} y \frac{\partial \psi}{\partial x}=\eta\left(\frac{\partial^{2} \psi}{\partial^{2} x}+\frac{\partial^{2} \psi}{\partial^{2} y}+\frac{\partial^{2} \psi}{\partial^{2} z}\right)
$$

with initial condition $\psi(x, y, t=0)=\psi_{0} \sin k_{0} x$, which has no dependence on $y$. When $\eta=0$, the field is just advected, resulting in $\psi(x, y, t)=\sin k_{0}\left(x-U_{0}^{\prime} y t\right)$, i.e. an unbounded creation of smaller scales along $y$, the shear-wise direction. When $\eta \neq 0$, a dissipative cut-off is introduced. In this case, we can look for a solution of the form $\psi=\psi_{0} f(t) \sin k_{0}\left(x-U_{0}^{\prime} y t\right)$, which gives

$f(t)=\exp \left(-\eta k_{0}^{2}\left(t+\frac{U_{0}^{\prime}}{3} t^{3}\right)\right)$

hence, the initial field evolves according to

$\psi(x, y, t)=\psi_{0} \sin k_{0}\left(x-U_{0}^{\prime} t\right) \exp \left(-\eta k_{0}^{2}\left(t+\frac{U_{0}^{\prime 2}}{3} t^{3}\right)\right)$.

From the above solution, we find that, for $\eta \ll 1$, the mixing time scale associated with the shear flow is

$\tau_{\text {shear }} \propto\left(\eta U_{0}^{\prime 2} k_{0}^{2}\right)^{-1 / 3}$.

The associated dissipative length $l_{\mathrm{d}}$ is found from Eq. (2), by balancing the advection term, the second on its left, with the diffusion (along $y$ ) term, the second on its right, $U_{0}^{\prime} l_{\mathrm{d}}(\psi / L) \sim$ $\eta\left(\psi / l_{\mathrm{d}}^{2}\right)$ :

$l_{\mathrm{d}} \sim\left(\frac{\eta L}{\boldsymbol{U}_{0}^{\prime}}\right)^{1 / 3}$

By performing a formal spectral transformation of Eq. (2) it is also found that individual modes evolve according to

$\frac{\partial \widehat{\psi}_{k}}{\partial t}+\boldsymbol{U}_{0}^{\prime} k_{y} \frac{\partial \widehat{\psi}_{k}}{\partial k_{x}}=-\eta\left(k_{x}^{2}+k_{y}^{2}\right) \widehat{\psi}_{k}$,

with the right hand side of the above expression representing advection in $k$-space. This also indicates that a shear flow results in a spectral expulsion towards high absolute-value shearwise wave numbers, corresponding to an increased tilting of the phase front in the flow direction. Thus, the physics behind the accelerated diffusion of the tracer $\psi$ in a shear flow is intuitively analogous to the one of phase mixing, which occurs for $\boldsymbol{V}_{\mathrm{A}}=\left(V_{\mathrm{A}}^{\prime} y, 0,0\right)$. To gain further insight into the problem, let us transform the advection-diffusion equation by introducing the "shearing coordinates" that follows the streamlines:

$\left[x^{\prime}=x-U_{0}^{\prime} y t, y^{\prime}=y, t^{\prime}=t\right]$.

From the chain rule for derivatives

$\left[\frac{\partial}{\partial x}=\frac{\partial}{\partial x^{\prime}}, \frac{\partial}{\partial y}=-U_{0}^{\prime} t \frac{\partial}{\partial x}+\frac{\partial}{\partial y^{\prime}}, \frac{\partial}{\partial t}=-U_{0}^{\prime} y \frac{\partial}{\partial x}+\frac{\partial}{\partial t^{\prime}}\right]$,

the advection-diffusion is now described by

$\frac{\partial \psi}{\partial t}=\eta\left(\frac{\partial^{2}}{\partial^{2} x}+\left(-U_{0}^{\prime} t \frac{\partial}{\partial x}+\frac{\partial}{\partial y^{\prime}}\right)^{2}\right) \psi$.

Keeping only the dominant terms in this equation, leads, after introduction of the new variable $\tau=\left(U_{0}^{\prime 2} t^{3}\right) / 3$, to a diffusion equation:

$\frac{\partial \psi}{\partial \tau}=\eta \frac{\partial^{2} \psi}{\partial^{2} x}$.

As is well known, there are two self-preserving solutions of a diffusion equation: harmonic and Gaussian. Their temporal evolution is different, however, their damping time is identical. Indeed, contrary to the harmonic solution studied above, an initial Gaussian packet has amplitude that decays as $1 /(\eta \tau)^{1 / 2}$, while it spreads. For the phase-mixing problem, such power-law behavior of localized initial wave-packets, i.e. its diffusion along field lines, was noticed and studied by Hood et al. (2002); see also Tsiklauri et al. (2003).

Now let us consider passive advection in a simple strain $\boldsymbol{U}=$ $(-\alpha x, \alpha y, 0)$ with a harmonic initial condition independent of $z$ :

$\frac{\partial \psi}{\partial t}-\alpha x \frac{\partial \psi}{\partial x}+\alpha y \frac{\partial \psi}{\partial y}=\eta\left(\frac{\partial^{2} \psi}{\partial^{2} x}+\frac{\partial^{2} \psi}{\partial^{2} y}+\frac{\partial^{2} \psi}{\partial^{2} z}\right)$.

A solution of the form

$\psi=\psi_{0} f(t) \sin \left(k_{x}(t) x+k_{y}(t) y\right)$

is sought, hence,

$\dot{k_{x}}=k_{x} \alpha ; \quad \dot{k_{y}}=-k_{y} \alpha$,

$k_{x}(t)=k_{0} \exp \alpha t ; \quad k_{y}(t)=k_{0} \exp -\alpha t$,

$\dot{f}=-\eta\left(k_{x}^{2}+k_{y}^{2}\right) f$ 
$\left.f(t)=\exp \left[-\frac{\eta k_{0}^{2}}{2 \alpha}\left(\exp ^{2 \alpha t}-1\right)\right)\right]$.

The field amplitude has super-exponential decay in time. It follows that, for $\alpha>0$, the mixing time due to a strain $\tau_{\text {strain }}$ is reduced compared to $\tau_{\text {shear }}$, i.e.

$\tau_{\text {strain }} \propto(1 / \alpha) \ln \left(\alpha / \eta k_{0}^{2}\right)$.

The reason is that, for a strain, the wave vector increases $e x$ ponentially with time and not linearly. And we can anticipate that this situation corresponds to enhanced dissipation of Alfven waves in the presence of a magnetic X-point configuration with $V_{\mathrm{A}}=(\alpha x, \alpha y, 0)$. This result remains unchanged for the threedimensional stagnation flow $\boldsymbol{U}=(\alpha x, \alpha y,-2 \alpha z)$. The dissipative scale length for advection and diffusion to balance each other in Eq. (12), is given by

$l_{\mathrm{d}} \sim\left(\frac{\eta}{\alpha}\right)^{1 / 2}$.

The general problem of the mixing of a passive scalar, in arbitrary flows, can be approached from a WKB description (Antonsen 1996). This is done, for instance, by introducing a filter, $\widehat{\psi}(\boldsymbol{k}, \boldsymbol{x}, t)=\int \mathrm{d}^{3} x \exp \left[-\boldsymbol{x}^{2} / \lambda^{2}+\mathrm{i} \boldsymbol{k}\left(\boldsymbol{x}-\boldsymbol{x}^{\prime}\right)\right] \psi\left(\boldsymbol{x}^{\prime}, t\right)$, with support $\lambda \ll L, L$ being the length scale of the variation in $\boldsymbol{U}$. It is a local Fourier transform in $\boldsymbol{x}$. Applying this transformation to the advection-diffusion Eq. (1), we obtain

$D_{t} \widehat{\psi}=-\eta k^{2} \widehat{\psi}$

with total derivative $D_{t}=\partial_{t}+\dot{\boldsymbol{x}} \nabla+\dot{\boldsymbol{k}} \nabla_{k}$ and $k^{2}=k_{x}^{2}+k_{y}^{2}+k_{z}^{2}$. Equation (20), which describes transport in $(\boldsymbol{x}, \boldsymbol{k})$ space, is also known as the kinetic wave equation in plasma physics (Sagdeev \& Galeev 1969) and includes non-linear coupling terms on its right-hand side. It is equivalently obtained by using the Wigner distribution (Bastiaans 1979; Antonsen 1996). The respective characteristics are

$\dot{\boldsymbol{x}}=\boldsymbol{U}=\nabla_{k} H$

$\dot{\boldsymbol{k}}=-\nabla(\boldsymbol{k} \cdot \boldsymbol{U})=-\nabla H$

with $H=\boldsymbol{k} \cdot \boldsymbol{U}$. The general solution of the kinetic wave Eq. (20) can be expressed in terms of phase space trajectories $\xi\left(\boldsymbol{x}^{\prime}, t\right)$ and $\kappa\left(\boldsymbol{x}^{\prime}, \boldsymbol{k}^{\prime}, t\right)$ where, $\mathrm{d} \xi / \mathrm{d} t=\boldsymbol{v}(\xi, t)$ and $\mathrm{d} \kappa / \mathrm{d} t=$ $-\nabla \boldsymbol{v}(\xi, t) . \kappa$, and $\xi\left(\boldsymbol{x}^{\prime}, 0\right)=\boldsymbol{x}^{\prime}, \kappa\left(\boldsymbol{x}^{\prime}, \boldsymbol{k}^{\prime}, 0\right)=\boldsymbol{k}^{\prime}$. The solution is $\widehat{\psi}(\boldsymbol{x}, \boldsymbol{k}, t)=\int \mathrm{d}^{3} x^{\prime} \mathrm{d}^{3} k^{\prime} \widehat{\psi}\left(\boldsymbol{x}^{\prime}, \boldsymbol{k}^{\prime}, 0\right) \delta\left(\boldsymbol{x}-\xi\left(\mathbf{x}^{\prime}, t\right)\right) \delta(\boldsymbol{k}-$ $\left.\kappa\left(\boldsymbol{x}^{\prime}, \boldsymbol{k}^{\prime}, t\right)\right) \exp \left(-\eta \int_{0}^{t} \kappa^{2} \mathrm{~d} t^{\prime}\right)$.

The physical interpretation of the WKB approach to mixing is clear. It means that the advected field is decomposed into an ensemble of packets with a length scale, $\lambda$, much smaller than the one, $L$, characterizing the spatial variation in the flow $\boldsymbol{U}$. Therefore, each packet experiences, along its trajectory, a velocity field $\boldsymbol{U}$ that is a linear function of the space coordinates: $\boldsymbol{U}\left(\boldsymbol{x}_{\mathrm{p}}(t)\right)+\partial \boldsymbol{U} /\left.\partial \boldsymbol{x}\right|_{\boldsymbol{x}_{\mathrm{p}}}(t) \cdot \boldsymbol{x}, \boldsymbol{x}_{\mathrm{p}}(t)$ being the position of the packet at time $t$. An important quantity, therefore, is the local rate of strain tensor, $\partial \boldsymbol{U} / \partial \boldsymbol{x}$, of the flow. To sum up, the packets are only deformed by the shear and the strain considered below. This brings us to the analogous effect for Alfven wave packets.

\section{Mixing of shear Alfven waves}

The ray equations of optics are $\dot{\boldsymbol{x}}=\nabla_{k} \omega$ and $\dot{\boldsymbol{k}}=-\nabla \omega$, with $\omega(\boldsymbol{k})$ the wave dispersion relation. The eikonal description of MHD waves was introduced by Weinberg (1962); see also Similon \& Sudan (1989), Petkaki et al. (1998), who used it in the context of wave dissipation in the corona. For the non-dispersive shear Alfven waves, with $\omega= \pm \boldsymbol{k} \cdot \boldsymbol{V}_{\mathrm{A}}$, the ray equations read

$\dot{x}= \pm V_{\mathrm{A}}$,

$\dot{\boldsymbol{k}}=\mp \nabla\left(\boldsymbol{k} \cdot \boldsymbol{V}_{\mathrm{A}}\right)$,

with the energy equation being

$\dot{e}_{ \pm}=-\eta k^{2} e_{ \pm}$,

this is required to study the energetics of the mixing process. The equation of motion, Eq. (23), states that shear-Alfven wave packets propagate along magnetic field lines so they disperse as the field lines do. It provides information on the precise path along which energy is deposited. The enhanced dissipation time follows from Eq. (24), giving the stretching rate of wave packets, i.e. the variation in their wave-vector, when combined with the energy equation. Dispersion and stretching are related, however, as it follows from consideration of two infinitesimally separated trajectories and the phase gradient, $\nabla \psi \equiv \boldsymbol{k}$, they generate in an inhomogeneous Alfvenic flow $\boldsymbol{V}_{\mathrm{A}}$. Indeed, constancy of the phase along a trajectory implies that $\psi(\xi(\boldsymbol{x}+\boldsymbol{r}, t), t)-\psi(\xi(\boldsymbol{x}, t), t)=\delta \xi(\boldsymbol{x}, t) . \nabla \psi(\xi(\boldsymbol{x}, t), t)=$ const. with $\delta \xi(\boldsymbol{x}, t)=\delta \xi(\boldsymbol{x}+\boldsymbol{r}, t)-\delta \xi(\boldsymbol{x}, t)$ being the differential separation vector. Thus, $\mathrm{d}(\delta \xi \cdot \boldsymbol{k}) / \mathrm{d} t=0$. According to Eq. (23), the trajectory of a wave packet is $\dot{\xi}= \pm \boldsymbol{V}_{\mathrm{A}}(\xi, t)$ and therefore $\mathrm{d} \delta \xi(\boldsymbol{x}, t) / \mathrm{d} t= \pm \delta \xi \cdot \nabla \boldsymbol{V}_{\mathrm{A}}(\xi(\boldsymbol{x}, t), t)$. Hence, the stretching properties of the Alfvenic flow, represented by Eq. (24) for the wave vector are related to the dispersion of magnetic field lines. Considering the phase mixing situation, with an Alfvenic shear flow $\boldsymbol{V}_{\mathrm{A}}=V_{\mathrm{A}}^{\prime} y \boldsymbol{x}, \mathrm{d} k_{y} / \mathrm{d} t=k_{x} V_{\mathrm{A}}^{\prime}$ with solution $k_{y}(t)=k_{x} V_{\mathrm{A}}^{\prime} t$ and integrating the energy equation, the typical time scale for the phase mixing is found to indeed correspond to $\tau_{\text {shear }}$, i.e.,

$\tau_{\text {mix }} \propto S^{1 / 3} \ll S$

with $S$ the Lundquist number $S \gg 1$. In the same way, the component of the wave vector in the direction of contraction $\boldsymbol{x}$ increases now exponentially with time, i.e. $\boldsymbol{k}_{x}(t)=k_{0} \exp (\alpha t)$, for the enhanced dissipation of shear Alfven waves at a magnetic X-point, which corresponds to a an Alfvenic strain flow $\boldsymbol{V}_{\mathrm{A}}=\alpha x \boldsymbol{x}-\alpha y \boldsymbol{y}, \alpha \equiv V_{\mathrm{A}}^{\prime}$. Integrating the energy equation results in the above-mentioned superexponential decay, so the enhanced dissipation time corresponds to $\tau_{\text {strain }}$

$\tau_{\text {mix }} \propto \ln S \ll S^{1 / 3}$.

This dependence of the dissipation time on the Lunquist number for the Alfvenic strain is also typical of wave damping in complicated magnetic geometries, either if the magnetic field is stochastic (Similon \& Sudan 1989) or regular, but possesses separators (Malara et al. 2003, 2007). The physics is the same however, and is related to the divergence of nearby magnetic field lines. In all these cases, the dissipation time of a wave packet depends weakly on the Lunquist number only as a logarithm, $\tau_{\text {mix }} \propto \ln S$, and not as a power law, $\tau_{\text {mix }} \propto S^{1 / 3}$ obtained for a unidirectional magnetic field, i.e. standard phase mixing. It is now clear that the propagation of shear Alfven waves in an inhomogeneous magnetic field is equivalent to a mixing process. Since the kinematics of mixing are governed by the topological properties of the flow, it turns out that the enhanced dissipation of Alfven waves propagating in a chaotic magnetic field is equivalent to a chaotic mixing process, from an energetics viewpoint. The spectral property of the wave energy flux in this linear process is the subject of the next section. 


\section{Wave energy spectrum in chaotic magnetic fields}

Since the global wave perturbation can be treated within the eikonal formulation as a superposition of wave packets with wave-vector $\boldsymbol{k}_{j}$, each carrying an energy $e_{j}(t)$, we can also write the wave-energy spectrum in the form:

$$
F(k, t)=\sum F_{j}(k, t)=\sum e_{j}(t) \delta\left(k-\left|\boldsymbol{k}_{j}(t)\right|\right) .
$$

In the presence of a source of wave energy, continuous shearing/straining experienced by wave packets along their trajectory leads to a cascade of their energy, hence of the energy of the global wave perturbation. This cascade can be studied in two different ways. In the first, there is no steady source, but only an initial distribution of wave packets and energy in space. For this initial value problem, the interest is in the subsequent transient evolution of the wave energy spectrum $F_{\mathrm{I}}(k, t)$ with initial condition $F_{\mathrm{I}}(k, t=0)=\sum e_{j}(t=0) \delta\left(k-\left|\boldsymbol{k}_{j}(t=0)\right|\right)$. As time increases, each wave number $k_{j}$ increases in time (on average), evolves toward the dissipation range, so that the wave energy decays. A second approach consists in taking into account the continuous injection of wave energy given by the ongoing perturbations. Therefore, wave packets and energy are supposed to be continually injected at intermediate $k$ values, around $k_{0}$, a typical injection scale, with $L^{-1} \ll k_{0} \ll k_{\mathrm{d}}$ and with $k_{\mathrm{d}}$ the very small dissipative cutoff and $L$ the global length scale associated with the magnetic field. In this case, electromechanical energy injection, say the photospheric drive, becomes balanced by heating and dissipation at high $k$, and a time-averaged stationary spectrum $F_{\mathrm{S}}(k)$ builds up.

We now turn to the study of the wave energy spectrum that results from quasi-uniform Alfvenic strain experienced by wavepackets as they propagate in a field with destroyed magnetic surfaces, i.e. along chaotic magnetic field lines. This configuration, which is a paradigm in transport theory for the calculation of anomalous diffusion coefficients (Rechester \& Rosenbluth 1977), was first considered in the context of coronal wave dissipation by Similon \& Sudan (1989), although they were only interested in the mixing time scale. Calling $\varepsilon$ the input wave energy flux, dimensional analysis yields

$\varepsilon \propto V_{\mathrm{A}}^{\prime} k F_{\mathrm{S}}(k)$,

with $F_{\mathrm{S}}(k)$ the stationary wave energy spectrum. Hence,

$F_{\mathrm{S}}(k) \propto k^{-1} V_{\mathrm{A}}^{\prime-1} \varepsilon$,

in the range where dissipation is negligible, i.e. for $k<k_{\mathrm{d}}=$ $\left(V_{\mathrm{A}}^{\prime} / \eta\right)^{1 / 2}$. This relation (30) for the wave energy, which is again derived below from a kinetic equation, is the same as the Batchelor's spectrum (Batchelor 1959) for scalar variance, obtained for a flow with uniformly distributed rate of strain, i.e. with chaotic streamlines.

Another derivation of (30) follows from writing down the evolution equation for the wave energy spectrum $F(k, t)$ which is obtained by taking the time derivative of Eq. (28):

$\frac{\partial F(k, t)}{\partial t}=-\eta k^{2} F(k, t)+\frac{\partial}{\partial k}\left[V_{\mathrm{A}}^{\prime} k F(k, t)\right]$.

The first term on the right hand side represents the effect of dissipation, $\dot{e}=-\eta k^{2} e$, and vanishes for $\eta=0$. The second term describes transport in $k$-space for wave packets that experience a uniform rate of strain, $V_{\mathrm{A}}^{\prime}=$ const., i.e. $\dot{k}(t)=V_{\mathrm{A}}^{\prime} k$. The stationary solution of (31) for $\eta=0$ is again $F_{\mathrm{S}}(k) \propto k^{-1}$. For $\eta \neq 0$, the stationary solution to Eq. (31), with energy source $\epsilon$ added at wave numbers where wave energy is fed into the system, is

$F_{\mathrm{S}}(k)=\frac{\epsilon}{V_{\mathrm{A}}^{\prime} k} \exp \left(-\eta k^{2} / \alpha\right)$

This shows the existence of a Gaussian dissipative fall-off, for $F_{\mathrm{S}}(k)$, at large $k>k_{\mathrm{d}}$.

Let us note, that while the derivation of the kinetic Eq. (31) is based on the WKB approximation, the dimensional analysis obtention of the $k^{-1}$ stationary spectrum does not rely on the assumption of a separation of scales. The latter is only based the wave energy transfer time being a constant, independent of the scale and given by $V_{\mathrm{A}}^{\prime}$, the quasi-uniform rate of strain experience by the waves.

Finally, we turn back to the initial value problem for transient forcing. Discarding dissipation in Eq. (31) and changing to the new variables, $\xi=\ln (k)$ and $G=k F(k)$, we can write Eq. (31) as

$\frac{\partial G}{\partial t}=V_{\mathrm{A}}^{\prime} \frac{\partial G}{\partial \xi}$.

Therefore, this transport equation reveals that, if an input wave energy pulse, $F_{\mathrm{I}}(k, t=0)$, is initially centered on intermediate $k$ values, say $k=k_{0}$, it is then carried conservatively at constant speed $\alpha \equiv V_{\mathrm{A}}^{\prime}$, on a logarithmic scale, until it reaches the dissipative range $k=k_{\mathrm{d}}$. Only there, it does experience fast damping and finally convert its energy into heat. This spectral viewpoint also contains the time scale involved before dissipation occurs: it is the travel time in the energy conserving-range in $k$-space, $\tau=\left(\ln k_{\mathrm{d}}-\ln k_{0}\right) / V_{\mathrm{A}}^{\prime}=\left(2 / V_{\mathrm{A}}^{\prime}\right) \ln \left(V_{\mathrm{A}}^{\prime} / \eta k_{0}^{2}\right) \propto \ln S$, which is $\tau_{\text {strain }}$.

Before concluding, a few comments are due. First, let us note that the magnitude of the energy flux $\epsilon$ depends on the amplitude of the drive, but not on its frequency, see e.g. Velli (2003). However, the location of the energy source in $k$-space depends on the range of driving frequencies. Recall that, since the dynamical equations are linear, consideration of a broad frequency drive does not alter the shape of the spectrum in the gap between the largest input wavenumber and the dissipative one. The very existence of such conservative range with neither source nor dissipation is suggested by the following reasoning. Since the wave number associated with the dissipative scale is very high, $k_{\mathrm{d}}=\left(V_{\mathrm{A}}^{\prime} / \eta\right)^{1 / 2} \gg 1$, the condition that the latter is also much greater than the highest input wave number $k_{\| \max }=\omega / V_{\mathrm{A}}$; i.e. $k_{\| \max } \ll k_{\mathrm{d}}$, is equivalent to $\omega \tau_{\mathrm{A}} \ll L\left(V_{\mathrm{A}}^{\prime} / \eta\right)^{1 / 2} \sim S^{1 / 2}$. This condition can easily hold for waves with $\omega \tau_{\mathrm{A}} \gg 1$ or equivalently, $k_{\|} \gg L^{-1}$, for which the WKB approximation holds. In their original work, Similon \& Sudan (1989), were able to relax the condition $k_{\|} \gg L^{-1}$ making it possible to study the behavior of the lower frequency fluctuations and non-WKB effects. Their tactic is entirely analogous to the reduced magnetohydrodynamics (RMHD) approximation, although they refer to the ballooning representation in fusion studies. By doing so, they only restrict the eikonal representation to the perpendicular direction. Low- frequency motion at the boundary of a unidirectional magnetic field is well known for producing turbulence and an energy cascade leading to a power-law energy spectrum (see for instance two recent works, Rapazzo et al. 2007; Dmitruk et al. 2003, and references therein). For realistic forcing (i.e. small amplitudes of the Alfven waves that are launched into the corona, $\delta u \sim 10^{-3} V_{\mathrm{A}}$; Acton et al. 1981), numerically obtained spectra are generally found to be quite steep. The reason resides in the weakness of the nonlinear interactions. However, since the 
background's large-scale field is taken as a constant, only the non-linearity can there provide the necessary transfer of energy on small dissipative scales. The linear mixing process with its associated energy flux is likely to alter energy transfer rates and hence spectra in inhomogeneous magnetic backgrounds. This is suggested by the numerical studies by Malara et al. (1992) and Einaudi et al. (1996), who showed that scattering of Alfven waves with the background inhomogeneity, for a population of waves initially propagating, say, upward, can act as a source for downward-propagating waves, so also as a trigger for the occurrence of non-linear interactions. We did not consider such effect in the present work and we are not aware of any MHD turbulence theory that accounts for how the linear and non-linear mechanisms of energy flux combine together. We find this subject an interesting area for further research.

We finally mention that the mixing effect considered in the present article, is the root of a proposed geometrical interpretation for the consequence of non-linear interactions among counter-propagating wave packets. It is clearly illustrated by Fig. 1 in Maron \& Goldreich (2001). Indeed, take a sample of originally straight vertical field lines which are perturbed by, say, downward-propagating waves. As a consequence, the field lines wander around each other. Therefore, if a localized wave packet is launched and propagates upward in this braided field, it will be distorted [also compare with Fig. 2 in Similon \& Sudan (1989) or the figure in Rechester \& Rosenbluth (1977)]. After several of these collisions, dissipation takes over and the wave packet mixes in its environment.

\section{Conclusions}

In this work, we consider the wave energy flux on small scales that results from the distortion of Alfven waves propagating in an inhomogeneous mean magnetic field $\boldsymbol{B}_{0}$. This problem is related to turbulence because the existence of a mean field may provide separation between linear and non-linear time scales. In an inhomogeneous magnetic field, Alfven wave packets disperse and are stretched by an Alfvenic flow $\boldsymbol{V}_{\mathrm{A}}=\boldsymbol{B}_{0} /\left(\mu_{0} \rho\right)^{1 / 2}$, whose streamlines are also the magnetic field lines. In a plasma with uniform density $\rho$, this flow is incompressible, i.e. $\nabla \cdot \boldsymbol{V}_{\mathrm{A}}=0$, and its stretching properties are thus only related to magnetic field lines dispersion. While the wave packets transport their energy at the group velocity $\boldsymbol{V}_{\mathrm{A}}$, so that while they disperse in physical space as the field lines do, they also experience continuous shearing and straining; i.e., they tend to mix. This gives rise to a cascade of the wave energy in $k$-space. As is well known for mixing, the efficiency of stretching relies on the local exponential separation of nearby trajectories (Ottino 1988). Since for a continuous external wave energy drive, the cascade of this energy ultimately leads to a balance between source and heating in the dissipative range, a stationary wave energy spectrum can build up even in the absence of significant non-linear interactions among the waves, a phenomenon that is absent in a constant mean magnetic field. Assuming the existence of a conservative range, we determined the wave-energy spectrum for the special case of wave packets propagating in a fully chaotic magnetic field. It means that the distribution of Lyapunov exponents, representing the rate of local exponentiation of nearby field lines or equivalently the degree of stretching of wave packets is strongly peaked at an average value $\alpha$. This situation, which was first considered by Similon \& Sudan (1989), for dissipation of Alfven waves corresponds to chaotic mixing by flows with uniformly a distributed rate of strain (Batchelor 1959; Antonsen et al. 1996).

Acknowledgements. This work was supported by the Science and Technology Facilities Council (STFC).

\section{References}

Acton, L. W., Culhane, J. L., Wolfson, C. J., et al. 1981, ApJ, 224, L137

Antonsen, T., Fan, Z., Ott, E., \& Garcia-Lopez, E. 1996, Phys. Fluids, 8, 3094

Bastiaans, M. J. 1979, Opt. Acta, 26, 1333

Batchelor, G. K. 1959, J. Fluid Mech., 143, 1

Browning, P. 1991, Plasma Phys. Control. Fusion, 33, 539

Dmitruk, P., Gomez, D. O., \& Matthaeus, W. H. 2003, Phys. Plasmas, 10, 3584

Einaudi, G., Califano, F, \& Chiuderi, C. 1996, ApJ, 472, 853

Elsasser, W. M. 1950, Phys. Rev., 79, 183

Heyvaerts, J., \& Priest, E. R. 1983, A\&A, 117, 220

Hood, A. W., Brooks, S. J., \& Wright, A. N. 2002, Proc. Roy. Soc Lond., 458, 2307

Malara, F., Veltri, P., Chiuderi, C., \& Einaudi, G. 1992, ApJ, 396, 297

Malara, F., Petkaki, P., \& Veltri, P. 2000, ApJ, 533, 523

Malara, F., De Francheschis, M. F., \& Veltri, P. 2003, A\&A, 412, 529

Malara, F., De Francheschis, M. F., \& Veltri, P. 2007, A\&A, 467, 1275

Maron, J., \& Goldreich, P. 2001, ApJ, 554, 1175

Ofman, L. 2005, Sp. Sci. Rev., 120, 67

Ottino, J. M. 1988, The Kinematics of Mixing: stretching, Chaos, and Transport (Cambridge: Cambridge University Press)

Petkaki, P., Malara, F., \& Veltri, P. 1998, ApJ, 500, 483

Rappazzo, A. F., Velli, M., Einaudi, G., \& Dahlburg, R. B. 2007, ApJ, 657, L47

Rechester, A. B., \& Rosenbulth, M. N. 1977, Phys. Rev. Lett., 40, 38

Ruderman, M. S., Nakariakov, V. M., \& Roberts, B. 1998, A\&A, 338, 1118

Tsiklauri, D., Nakariakov, V., \& Rowlands, G. 2003, A\&A, 400, 1051

Similon, P. L., \& Sudan, R. N. 1989, ApJ, 336, 442

Smith, P., Tsiklauri, D., \& Ruderman, M. S. 2007, A\&A, 475, 1111

Sagdeev, R., \& Galeev, A. 1969, Nonlinear Plasma Theory (New-York: Benjamin)

Velli, M. 2003, Plasma Phys. Control. Fusion, 45, 205

Walsh, R. W., \& Ireland, J. 2003, A\&ARv, 12, 1

Weinberg, S. 1962, Phys. Rev., 126, 1899 